\title{
SUNLIGHT AND HYPERBILIRUBINEMIA: A SYSTEMATIC REVIEW
}

\author{
Maki Zamzam1), Lidia Ekiq Kurniavie²) \\ 1)Faculty of Public Health, Universitas Airlangga \\ ${ }^{2)}$ Masters Program in Public Health, Universitas Sebelas Maret
}

\begin{abstract}
Background: Babies with jaundice are often treated with phototherapy lamps, which emit blue-green light that alters the bilirubin (yellow substance found naturally in the baby's blood) so that it can be more easily excreted. Sunlight emits light in a similar spectrum. This study aimed to review systematically the relative efficacy of sunlight in comparison with phototherapy unit for the reduction of bilirubin concentration in neonates with jaundice.

Subjects and Method: This was a systematic review. The research question formulated in PICO was as follows. Population: newborn baby. Intervention: sunlight with or without filtering. Comparison: phototherapy. Outcome: bilirubin concentration. The primary study articles between 2016-2020 were searched from databases including PubMed, Wiley, Science Direct, and Cochrane. The inclusion criteria was research articles published fulltext in English. The keywords were "sunlight" and "hyperbilirubinemia". The data were reported systematically using PRISMA.

Results: A total of 5 research articles from USA, Africa, and India met the inclusion criteria and included in this review. Sunlight with or without filtering was more effective than a phototherapy unit to reduce bilirubin concentration in neonates with jaundice.

Conclusion: Sunlight with or without filtering is more effective than a phototherapy unit to reduce bilirubin concentration in neonates with jaundice.
\end{abstract}

Keywords: sunlight, hyperbilirubinemia.

Correspondence:

Maki Zamzam. Univeristas Airlangga. Surabaya, East Java, Indonesia. Email: makizz477@gmail.com. Mobile: +6285852540575. 